\title{
Susceptibilidad de híbridos de girasol (Helianthus annuus) a la podredumbre media del tallo y quebrado caulinar producido por Sclerotinia sclerotiorum
}

\author{
Gabriela Reimonte y Fernando Castaño ${ }^{1}$ \\ Mejoramiento Genético Vegetal, Facultad de Ciencias Agrarias, Universidad Nacional \\ de Mar del Plata (UNMdP), Unidad Integrada Balcarce, RN Nº 226, Km 73,500, CC 276, B 7620 BKL, \\ Balcarce, Argentina.
}

\begin{abstract}
G. Reimonte, and F. Castaño. 2008. Susceptibility of sunflower hybrids (Helianthus annuus) to mid stem rot and broken stem caused by Sclerotinia sclerotiorum. Cien. Inv. Agr. 35(1):27-35. In the sunflower, Sclerotinia sclerotiorum is the cause of stem rot and broken stems. Seventeen sunflower hybrids were evaluated in Balcarce, Argentina after mid-stem inoculation. The association between rot length and the rate of stem breakage was calculated. Sunflower hybrids showed considerable differences in the size of the rot lesion developed and in the relative number of broken stems, as determined 20 days after the beginning of flowering (daf). No variability was detected for broken stems, both 6 and 60 days after inoculation. Sunflower plants with greater mid stem rot length had a greater relative number of broken stems. The incidence of broken stems could have been enhanced by the mid-stem rot, especially in those genotypes with the lowest proportion of structural tissues in the stems. The degree of genetic determination suggested that environmental effects influence the length of symptoms less than that the proportion of broken stems determined at 20 daf. The results allowed us to propose a breeding program based on the selection of the most adequate genotypes given their level of resistance to these two characters. A phenotypic selection could be performed on individual plants based on the extent of rotting caused by S. sclerotiorum at the mid-stems. Then, the proportion of broken stems could be scored on F2-derived lines in F3 (F2:3 line).
\end{abstract}

Key words: Breeding, broken stem, disease resistance, Helianthus annuus, Sclerotinia sclerotiorum, stem rot.

\section{Introducción}

El girasol (Helianthus annuus L.) tiene interés agronómico y gastronómico debido a la alta calidad nutricional de su aceite. En Argentina, casi las tres cuartas partes del aceite consumido provienen de esta planta oleaginosa. Además, tiene un alto interés económico por los ingresos de divisas generados por las exportaciones de granos, aceite y subproductos del girasol (Ingaramo, 2006).

Sclerotinia sclerotiorum (Lib.) de Bary es un hongo ascomicete, muy polífago, con más

Recibido 19 julio 2007. Aceptado 13 noviembre 2007.

1Dirigir correspondencia a F. Castaño: fcastanio@balcarce.inta.gov.ar de 360 especies hospederas. Entre éstas, el girasol es un importante hospedero, susceptible a las infecciones de este patógeno durante casi todo su ciclo de vida. Son susceptibles los cotiledones, el botón apical, la base del tallo, las hojas y el capítulo. Sin embargo, los ataques sobre capítulos causan las mayores pérdidas en Argentina, Europa y EUA (Gulya et al., 1997). Además, los daños ocasionados en la parte media del tallo, como resultado de una infección foliar, son asimismo muy perjudiciales. En Canadá (Dorrel y Huang, 1978), Francia (Pérès et al., 1991) y Argentina (Sala et al., 1994) han ocurrido pérdidas entre 20 y $70 \%$ del rendimiento, las que se han atribuido a la interrupción parcial o total de la nutrición de la inflorescencia y/o por la reducción del número de capítulos cosechados 
como consecuencia de plantas quebradas por la acción de este patógeno.

El uso de híbridos resistentes es una de las estrategias propuestas para controlar la podredumbre media del tallo (Castaño et al., 2001a, 1991). La evaluación y selección de genotipos resistentes a $S$. sclerotiorum se puede realizar en condiciones de infección natural. Utilizando esta metodología, se han detectado comportamientos diferenciales y una relación entre el largo de la lesión del tallo con el contenido de aceite y el número de granos en seis híbridos experimentales evaluados en Argentina (Sala et al., 1994). Sin embargo, la aparición natural de la podredumbre caulinar es errática. La infección depende de factores ambientales (ej. ambientes frescos y húmedos) durante la infección foliar, así como de un nivel de resistencia foliar y peciolar relativamente bajos, de modo que el patógeno pueda invadir el tallo a partir de la infección foliar o peciolar.

Degener et al. (1998) evaluaron la variabilidad de las respuestas de 85 líneas de girasol de Alemania y EUA a la infección del tallo, utilizando el protocolo francés de inoculación en hojas (Bertrand y Tourvieille, 1987). Micic et al. (2005) detectaron tres QRL's (Loci de resistencia cuantitativa que controlan resistencias horizontales) relacionados con la podredumbre del tallo. Realizando inoculaciones directamente sobre la parte media del tallo, Castaño et al. $(1993,1989)$ cuantificaron la longitud de la podredumbre y evaluaron la variabilidad de la lesión del tallo en una serie de líneas de girasol francesas.

Los objetivos de este trabajo fueron estudiar la asociación entre el quebrado caulinar producido por $S$. sclerotiorum y la resistencia de híbridos de girasol, en inoculaciones realizadas en la parte media del tallo.

\section{Materiales y métodos}

\section{Híbridos de girasol}

Se utilizaron 17 híbridos de girasol (Cuadro 1). De éstos, 13 fueron de Argentina (Aca 882, Contiflor 9, Dekalb G100, Dekasol 3881, Morgan 704, Morgan 738, Norkin Punta,
Paraíso 4, Pioneer 6440, Rancul, TC 3003, Tresur Naciente y Zénit). Estos cultivares se eligieron al azar de una lista de cultivares sembrados en Argentina a fines de la década los años noventa. El híbrido TC 3003 aún se recomienda para las regiones del centro y del sur de la Provincia de Buenos Aires (Asagir, 2006).

\section{Inoculación}

En función de resultados previamente obtenidos (Castaño et al., 2001b, 1992), se obtuvo micelio a partir de esclerocios de $S$. sclerotiorum en agar-papa glucosado al 2\% (APG). Los cultivos se incubaron por tres días en oscuridad a $23 \pm$ $1^{\circ} \mathrm{C}$.

Se emplearon plantas en el estadio de desarrollo R4 (Schneiter y Miller, 1981) (=E4, Anónimo, 1992). Esta etapa ocurrió alrededor de siete días antes del inicio de la floración. Cada planta se inoculó con un trozo de micelio (5 $\mathrm{mm}$ de diámetro), obtenido con la ayuda de un sacabocados, desde el margen de las colonias de S. sclerotiorum desarrolladas en APG. El inóculo se depositó sobre el tallo previamente herido, raspando la cutícula, en un entrenudo del tercio superior del tallo, aproximadamente un metro desde el suelo. El sitio de inoculación se cubrió con papel de aluminio (150x100 $\mathrm{mm}$ ) para impedir el rápido desecamiento del inóculo.

\section{Evaluación del quebrado del tallo}

Se determinó la proporción de plantas cuyos tallos se rompieron a la altura de la zona de la inoculación al momento de medir la longitud de podredumbre y a los 20 [estadio R7 (=M0)] y 60 días desde el inicio de floración (dif) (madurez de cosecha, estadio M4) (Anónimo, 1992).

\section{Diseño, análisis estadísticos y genéticos}

Todos los híbridos se distribuyeron en el campo siguiendo un diseño en bloques completos al azar con dos repeticiones. Cada unidad experimental, consistió en 20 plantas en un surco de $5 \mathrm{~m}$ de largo por $0,70 \mathrm{~m}$ de ancho.

Se realizaron análisis de la varianza y los 
Cuadro 1. Susceptibilidad relativa de híbridos de girasol (Helianthus annuus) a Sclerotinia sclerotiorum, determinada por inoculaciones con micelio de $S$. sclerotiorum en la parte media del tallo.

Table 1. Relative susceptibility of the sunflower (Helianthus annuus) to Sclerotinia sclerotiorum, as determined by inoculations performed with mycelium of $\mathrm{S}$. sclerotiorum in the mid portion of the stems.

\begin{tabular}{|c|c|c|c|c|c|}
\hline \multirow[b]{2}{*}{ Híbridos } & \multicolumn{2}{|c|}{ Largo de lesión } & \multicolumn{3}{|c|}{ Plantas quebradas } \\
\hline & $\mathrm{mm}$ & $\begin{array}{l}\text { Nivel de } \\
\text { resistencia }{ }^{1}\end{array}$ & $\begin{array}{c}20 \mathrm{ddf}^{2} \\
\%\end{array}$ & $\begin{array}{l}\text { Nivel de } \\
\text { resistencia }{ }^{1}\end{array}$ & $\begin{array}{c}60 \mathrm{ddt}^{2} \\
\%\end{array}$ \\
\hline Rancul & 167 & bajo & 100 & bajo & 100 \\
\hline Aca 882 & 150 & bajo & 100 & bajo & 100 \\
\hline Tresur Naciente & 150 & bajo & 100 & bajo & 100 \\
\hline $74-\mathrm{F}$ & 147 & bajo & 100 & bajo & 100 \\
\hline Norkin Punta & 132 & medio & 100 & bajo & 100 \\
\hline Pioneer 6440 & 131 & medio & 94 & bajo & 100 \\
\hline Paraíso 4 & 114 & medio & 82 & bajo & 88 \\
\hline Contiflor 9 & 111 & medio & 82 & bajo & 94 \\
\hline Dekalb G100 & 108 & medio & 88 & bajo & 88 \\
\hline TC 3003 & 107 & medio & 94 & bajo & 100 \\
\hline Rémil & 99 & alto & 82 & bajo & 94 \\
\hline Morgan 704 & 98 & alto & 76 & bajo & 94 \\
\hline Zénit & 97 & alto & 100 & bajo & 100 \\
\hline Morgan 738 & 85 & alto & 54 & medio & 75 \\
\hline Dekasol 3881 & 84 & alto & 69 & bajo & 75 \\
\hline Euroflor & 80 & alto & 100 & bajo & 100 \\
\hline Sd-Pac1 & 69 & alto & 19 & alto & 69 \\
\hline Medias & $113 \pm 5,14$ & & $85 \pm 4,18$ & & 93 \\
\hline $\mathrm{CV}^{3}, \%$ & 14 & & 19 & & 16 \\
\hline $\mathrm{DMS}^{3}$ & 33 & & 34 & & ns \\
\hline
\end{tabular}

${ }^{1}$ Nivel de resistencia: bajo = valores estadísticamente no diferentes al valor máximo; medio = valores estadísticamente diferentes al valor máximo y mínimo, alto = valores estadísticamente no diferentes al valor mínimo.

${ }^{2}$ Días después de la floración (daf).

${ }^{3}$ Coeficiente de variación $(\mathrm{CV})$. Diferencia mínima significativa $(\mathrm{DMS})(\mathrm{p}=0.05)$. ns, análisis de varianza no significativo a $\mathrm{p}=0.05$.

${ }^{I}$ Levels of resistance: low $=$ statistically similar to maximum values, intermediate $=$ statistically different from maximum and minimum values, high= statistically similar to minimum values.

${ }^{2}$ Days after flowering (daf).

${ }^{3}$ Coefficient of variability $(C V)$. Least significant difference $(L S D)(p=0.05) . n$, analysis of variance not significant at $p=0.05$.

promedios se separaron de acuerdo con la prueba de diferencias mínimas significativas (DMS) (Steel y Torrie, 1988). De este modo los híbridos se clasificaron en tres grupos $(\mathrm{G})$ : G1, abarcó los materiales con baja resistencia, los que fueron estadísticamente similares ( $p$ $<0,05)$ a los híbridos que mostraron el valor máximo del carácter estimado; G2, estuvo integrado por los híbridos con resistencia alta, estadísticamente similares de aquéllos con los valores mínimos y G3, abarcó los híbridos no involucrados en G1 ni en G2 con resistencia intermedia.

Se determinó el grado de asociación lineal entre la longitud de podredumbre y la proporción de tallos quebrados por análisis de correlación lineal (Steel y Torrie, 1988).
Se estimó el grado de determinación genética $\left(\mathrm{GDG}=\mathrm{Vg} \cdot \mathrm{Vf}^{-1}\right)$ y su error estándar para establecer si la proporción de la variación fenotípica exhibida entre híbridos se explica genéticamente (Becker, 1985).

\section{Resultados}

\section{Podredumbre en la parte media del tallo}

Sin excepción (100\%), los tallos inoculados presentaron una podredumbre castaño claro a lo largo del tallo. Se observó decoloración de los tejidos y una abundante proliferación de micelio blanco y superficial con esclerocios internos negros. El raspado de la cutícula del tallo en el sitio de inoculación favoreció la infección y el desarrollo de la enfermedad (Cuadro 1). 
La longitud promedio de podredumbre a lo largo del tallo fue de $113 \pm 5,14 \mathrm{~mm}$. La variabilidad no controlada (CV) por el diseño experimental fue $14 \%$. El análisis de la varianza detectó respuestas altamente significativas $(\mathrm{p}=0,002)$ en los 17 híbridos evaluados (Cuadro 1).

En G1, los híbridos 74-F, Tresur Naciente y Aca 882 mostraron un tamaño de lesión similar al de Rancul, que exhibió el valor máximo (167 $\mathrm{mm})$. En G2 se agruparon los híbridos Sd-Pac1, con el valor mínimo de podredumbre en tallo (69 mm), así como también Euroflor, Dekasol 3881, Morgan 738, Zénit, Morgan 704 y Rémil. Finalmente, el G3 comprendió a los híbridos Norkin Punta, Pioneer 6440, Paraíso 4, Contiflor 9, Dekalb G 100 y TC 3003 (Cuadro 1).

\section{Quebrado de tallo en plantas enfermas}

Las plantas inoculadas no mostraron tallos quebrados a los seis días posinoculación. No obstante, estos síntomas se obtuvieron luego de 20 dif (Cuadro 1). La media general fue $85 \pm$ $4,18 \%$ y el CV $19 \%$. El análisis de la varianza reveló un efecto altamente significativo (p
$=0,007)$ entre híbridos a la quebradura del tallo. En el G1 se agruparon 15 híbridos, en los que ocho exhibieron similares valores de plantas quebradas que los híbridos con valores máximos (100\%: Rancul, Aca 882, Tresur Naciente, 74-F, Norkin Punta y Euroflor). Los híbridos Sd-Pac1 y Morgan 738 se ubicaron en G2 y G3, respectivamente. El híbrido Sd-Pac1 tuvo el valor mínimo en el experimento (19\%) (Cuadro 1).

Los valores de plantas quebradas, cuantificadas a los 60 dif, tuvieron en promedio $93 \pm 2,52 \%$ con un CV de 16\%. En esta última fecha de evaluación, el análisis de la varianza no fue significativo.

\section{Relación tamaño de podredumbre y plantas quebradas}

Una significativa correlación positiva se obtuvo entre las respuestas de los 17 híbridos de girasol a la podredumbre del tallo y al número relativo de plantas quebradas. A los 20 dif esta relación fue igual a $\mathrm{y}=0,504 \mathrm{x}+27,54(\mathrm{r}=0,66 ; \mathrm{p}=$ 0,003) (Figura 1).

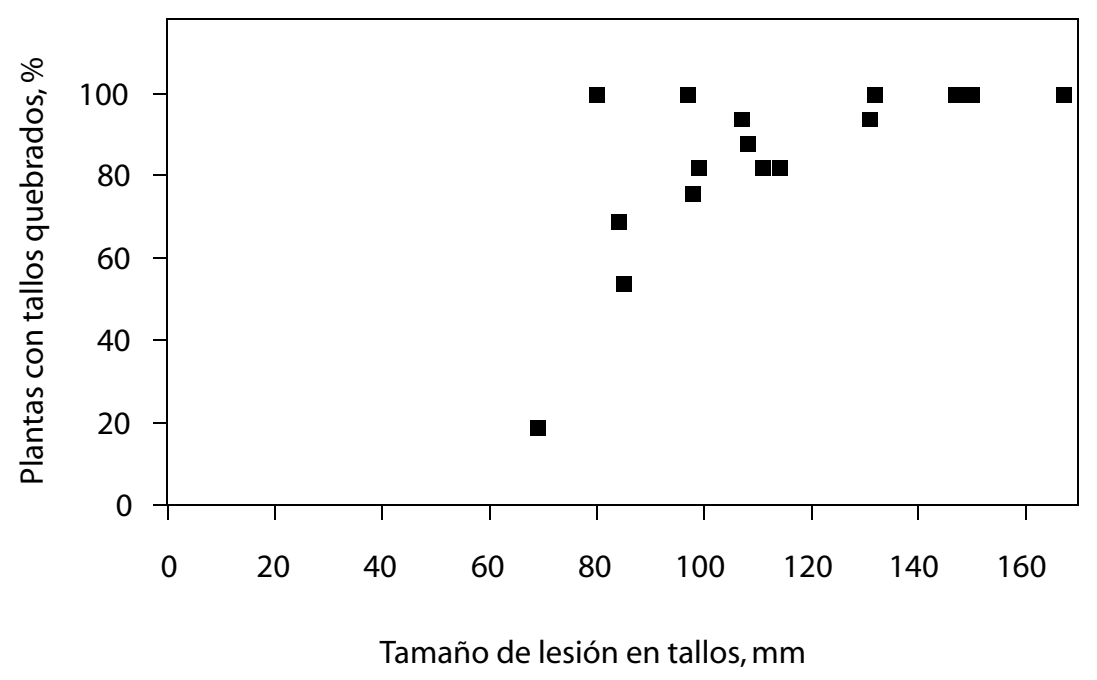

Figura 1. Relación entre la longitud de podredumbre en tallo y el quebrado caulinar del girasol (Helianthus annuus) producido por Sclerotinia sclerotiorum en híbridos de girasol luego de inoculaciones en el tallo empleando micelio y determinado luego de 20 días desde el inicio de la floración.

Figure 1. Relationship between the length of stem rot and the proportion of broken stems caused by Sclerotinia sclerotiorum in sunflower (Helianthus annuus) hybrids, after being inoculated with mycelium and evaluated 20 days after flowering. 


\section{Grado de determinación genética}

El grado de determinación genética (GDG) para la longitud de podredumbre en el tallo medio fue $0,85 \pm 0,07$, mientras que el GDG estimado para el porcentaje de tallos quebrados a los 20 dif fue $0,72 \pm 0,12$. Para ambos caracteres, estos valores sugieren que una alta proporción (casi las tres cuartas partes o más) de la variabilidad fenotípica expresada entre híbridos se debió a causas de origen genético.

\section{Discusión}

El protocolo de inoculación empleado en este trabajo fue exitoso para inducir los síntomas caulinares debidos a $S$. sclerotiorum, los que fueron coincidentes con los descritos previamente (Lamarque, 1985). El nivel de precisión con que se cuantificaron las respuestas al patógeno fue adecuada y similar a la obtenida con el mismo patógeno en otros órganos del girasol (Odriozola et al., 2006; Castaño y Legarreta, 2004).

De acuerdo con los resultados obtenidos, la longitud de la podredumbre fue independiente del diámetro del tallo, permitiendo evaluar cultivares con distintos grosoresenel eje caulinar (Castaño et al., 1989). Por otra parte, poder cuantificar la respuesta de los cultivares previo a la floración es de interés para el mejoramiento genético, porque es posible controlar los dos sexos cuando se desee entrecruzar las plantas seleccionadas. En este contexto, se aumentaría la eficiencia de selección, respecto de si dicho comportamiento se evaluase posfloración.

Si bien la aparición natural de la podredumbre en tallos ocurre como consecuencia de infecciones foliares de $S$. sclerotiorum, la evaluación del comportamiento de genotipos mediante inoculaciones en la parte media del tallo presentó ventajas respecto de la metodología propuesta por Degener et al. (1998). Se sabe que las respuestas a inoculaciones foliares y caulinares son independientes (Castaño et al., 1993). Por lo que un nivel de resistencia foliar moderadamente alto impediría detectar, mediante el método propuesto en otros trabajos, genotipos de pobre comportamiento a la inoculación caulinar (Micic et al., 2005; Degener et al., 1998).
La inoculación realizada permitió evidenciar la versatilidad del girasol a inoculaciones con S. sclerotiorum en la parte media del tallo. La variabilidad detectada en los cultivares (híbridos) evaluados en este trabajo, respecto de la longitud de podredumbre caulinar, coincidió con la diversidad señalada en híbridos experimentales y líneas endocriadas de esta planta oleaginosa (Degener et al., 1998; Sala et al., 1994).

En este experimento, Sd-Pac1 tuvo una mínima lesión (69 mm). Dicho híbrido, junto con 74-F, Euroflor y Rémil, son de origen francés y se utilizan en nuestro programa de mejoramiento, por su comportamiento a $S$. sclerotiorum y Phomopsis helianthi (Godoy et al., 2005; Castaño y Legarreta, 2004; García et al., 2000; Verschoor et al., 1998). El híbrido Sd-Pac1 se desarrolló en el INRA (Clermont Ferrand, Francia), a partir del cruzamiento entre las líneas Sd y Pacl. Estudios previos demostraron que cada una de estas retrazan el crecimiento del micelio en los órganos atacados por $S$. sclerotiorum (Castaño et al., 1993). La progenie híbrida de ambas líneas (ej. Sd-Pac1) se destacó en inoculaciones en capítulo y hojas (Castaño et al., 2005, 1992; Vear y Tourvieille, 1988). Por lo tanto, fue previsible que en este trabajo, el híbrido Sd-Pacl liderara el grupo de buen comportamiento frente a la podredumbre del tallo.

En girasol, el nivel de resistencia a las inoculaciones de $S$. sclerotiorum en hojas y capítulos se relaciona directamente con el contenido de fenoles preformados en ambos órganos de la planta (Prats et al., 2003; Castaño et al., 1992). Para la lesión foliar, el cultivar con el menor tamaño de podredumbre así como el mayor contenido de fenoles fue Sd-Pac1. Por lo tanto, es posible que este híbrido tenga un mecanismo de resistencia similar para la lesión del tallo.

Ocho de los 17 híbridos mostraron un número similar de plantas con tallos quebrados a los 20 y 60 dif. En efecto, los híbridos Rancul, Aca 882, Tresur Naciente, 74-F, Norkin Punta, Zénit y Euroflor tuvieron la máxima proporción esperable (100\%), mientras que Dekalb G100 mostró $88 \%$. El hecho que en la mayoría de los 
híbridos evaluados (53\%) la cantidad relativa de plantas quebradas continuó progresando después de los 20 dif justificó la realización de una tercera observación a los 60 dif.

Hubo variabilidad en la cantidad de plantas con tallos rotos en sólo una de las fechas de evaluación de este carácter. Por lo tanto, a los 20 dif algunos materiales mostraron mayor habilidad de permanecer erectos. En la primera fecha (6 días desde la inoculación) no hubo plantas quebradas. Mientras que, en la tercera (60 dif), el progreso de la proporción de tallos quebrados en la mayoría de las plantas del experimento motivó un acotamiento de la amplitud de variación del carácter y la consecuente pérdida de diversidad en las respuestas. Estos resultados sugieren que a los 20 dif sería un momento adecuado para detectar genotipos de buen comportamiento por cantidad relativa de plantas quebradas luego de la inoculación con S. sclerotiorum.

El análisis de correlación mostró una correspondencia relativamente elevada (dos tercios) y significativa entre la podredumbre caulinar y los tallos quebrados. Las plantas con menor tamaño de lesión en la parte media del tallo mostraron, generalmente, una proporción inferior de tallos quebrados y, viceversa. En este contexto, el híbrido Sd-Pacl tuvo la longitud mínima (69 mm) y el menor número relativo de tallos rotos (19\%). Esto provocó que se ubicara en el grupo de mayor nivel de resistencia (G2), para ambas variables. Mientras que, por el contrario, Rancul, Aca 882, Tresur Naciente y 74-F tuvieron todas sus plantas quebradas y los tamaños relativos mayores de lesión, por lo que se los colocó en el G1.

Sin embargo, hubo híbridos que se escaparon de esta generalización. Por ejemplo, Zénit y Euroflor mostraron una podredumbre poco extendida (G2) pero todas sus plantas quebradas a los 20 dif(G1). Knowles (1978) y Miller (1987) mencionaron que la capacidad del girasol a no quebrarse dependía de la robustez de su eje caulinar, tanto en presencia como en ausencia de infección parasitaria. Recientemente Sposaro et al. (2007) mostraron que la solidez y el sostén mecánico en plantas sanas del híbrido Zénit esta asociada con la delgadez de ciertos tejidos estructurales del tallo, corteza y epidermis. Dicha afirmación sugiere que, en nuestro trabajo, la presencia de $S$. sclerotiorum en el tallo de ese híbrido habría facilitado que alcance el límite extremo de plantas quebradas (100\%). En el híbrido Euroflor habría ocurrido una situación similar.

Las toxinas y enzimas de $S$. sclerotiorum provocan la desorganización y muerte de las células atacadas, debilitando los tejidos (Langar et al., 2002; Zimmer y Hoes, 1978). En forma similar, Fusarium moniliforme y Phytophthora capsici, deterioran y producen roturas en tallos en maíz y pimiento, respectivamente (Bartual et al., 1991; Foley, 1969). Por lo tanto, sería esperable que el quebrado del tallo se favorezca ante la presencia de $S$. sclerotiorum en tallos de cultivares con baja cantidad relativa de tejidos estructurales (ej. Zénit).

A la tercera fecha de evaluación no hubo variabilidad en el quebrado. Esto se relacionó con el incremento de plantas rotas en la mayor parte de los híbridos inoculados. Particularmente Sd-Pac1 registró el mayor aumento de tallos quebrados (260\%), por unidad de tiempo transcurrida desde los 20 dif. Durante este periodo (ej. 40 días), el mecanismo de defensa bioquímico y/o estructural de Sd-Pac1 habría permitido que algunas plantas permanecieran todavía erguidas.

Para la longitud de podredumbre, el valor de GDG sugirió que sólo el 15\% de la variabilidad fenotípica podría ser atribuida a causas meramente no genéticas. Los resultados de este estudio provienen de un sólo experimento, lo cual imposibilita conocer la interacción entre el genotipo y el ambiente (IGA). Esto tiene dos consecuencias: el valor de GDG $=85 \%$, aunque varias veces superior a su error estándar, podría estar sobrevalorado. Por otro lado, el comportamiento relativo de los híbridos en distintos ambientes frente a la inoculación se vería también afectado. Estas inconsistencias fueron también destacadas por Godoy et al. (2005) al discutir el efecto de la IGA para las infecciones de $S$. sclerotiorum en capítulos. Dichos autores mostraron una IGA del tipo cuantitativa o no-cruzada, debido a que el ranking de los genotipos no cambió al pasar de 
un ambiente a otro, y sugirieron que este tipo de interacción sería característica del patosistema en cuestión.

Lo anterior permite suponer que, para la podredumbre caulinar valorada en distintos ambientes, sería esperable un cambio en la magnitud del tamaño de lesión pero no una variación en la clasificación de los híbridos según su respuesta. La sobrevaloración del GDG sería una de las causas del por qué ese valor no concuerda con el de heredabilidad en sentido estricto $\left(h^{2}=0,59\right)$, calculado por Degener et al. (1999) para la podredumbre en tallo.

En este trabajo, el valor obtenido para la proporción de varianzas estimadas con los datos de podredumbre caulinar fue similar al calculado por Vear y Tourvieille (1988) para las inoculaciones ascospóricas de S. sclerotiorum en capítulos. Asimismo, es intermedio para otras formas de ataque del mismo patógeno: pruebas con micelio en capítulos (Castaño et al., 2001b; Robert et al., 1987), en hojas (Castaño et al., 1992) y en la base del tallo (Tourvieille y Vear, 1990).

Para la proporción de tallos quebrados a los 20 dif, el $72 \%$ de la diversidad fenotípica entre híbridos no puede ser atribuida a causas no genéticas. Lo anterior, permite afirmar que el ambiente afectaría más el quebrado caulinar que la longitud de la lesión luego de la inoculación con $S$. sclerotiorum.

Los resultados de este trabajo son también de interés en un programa de mejoramiento por resistencia. En este sentido, el relativamente bajo efecto del ambiente sobre la inoculación en tallo permitiría realizar una selección fenotípica por la extensión de la podredumbre caulinar al inicio de la floración del cultivo. Las plantas seleccionadas se deberían autofecundar y las semillas conservarse para ser sembradas a campo y evaluar las familias F3 derivadas en F2 (F2:3). Las semillas guardadas de las mejores familias seleccionadas proporcionarán las plantas que, a la temporada siguiente, serán entrecruzadas con el propósito de crear una población mejorada por ambos caracteres. Se deberá considerar la posibilidad de detectar productos bioquímicos relacionados con el mecanismo de defensa de la planta así como marcadores genéticos que brindarían, además, la posibilidad de evaluar el comportamiento en ausencia del patógeno.

Los resultados de este trabajo provienen de un sólo ensayo a campo. Por lo tanto, se hace necesario realizar experimentos adicionales, en otros años y regiones de Argentina, antes de establecer una conclusión definitiva. Sin embargo, es de interés destacar que, hasta donde es de nuestro conocimiento, esta es la primera vez que experimentalmente se relaciona el tamaño de la podredumbre con el quebrado caulinar producida por $S$. sclerotiorum en girasol.

\section{Resumen}

En el girasol (Helianthus annuus L.), cultivo de gran interés agronómico, Sclerotinia sclerotiorum ataca la parte media del tallo produciendo la podredumbre media del tallo. En Balcarce, Argentina, se realizó un ensayo a campo donde se evaluó la respuesta de 17 híbridos de girasol a $S$. sclerotiorum. Se evaluó la relación entre la lesión provocada por este patógeno y el quebrado del tallo enfermo. Los híbridos mostraron respuestas diferenciales al tamaño de la podredumbre y en el número relativo de tallos rotos, medido a los 20 días de iniciada la floración (dif). No hubo variabilidad de plantas quebradas a los 60 dif. Los híbridos con mayor longitud de lesión promedio mostraron mayor número de plantas quebradas. El debilitamiento del eje caulinar, particularmente en genotipos con menor proporción de tejidos de sostén, habría facilitado la rotura del tallo. El grado de determinación genética sugirió que la longitud de las lesiones estaría menos influenciada por factores ambientales que la proporción de plantas quebradas a los 20 dif. Estos resultados permiten delinear un programa de mejoramiento, para incrementar el nivel de resistencia a esta enfermedad en girasol, basado en la selección de genotipos para ambos caracteres. Una selección fenotípica se podría realizar sobre plantas individuales, utilizando el carácter extensión de la podredumbre en tallos. La proporción de plantas quebradas se evaluaría, luego, en las familias F3 derivadas en F2 (líneas F2:3). 
Palabras clave: Helianthus annuus, mejora genética, podredumbre caulinar, quebradura de tallo, resistencia, Sclerotinia sclerotiorum.

\section{Agradecimientos}

La autora realizó este trabajo como parte de los requisitos para obtener el título de Ingeniero Agrónomo, Facultad de Ciencias Agrarias, Universidad de Mar del Plata (UNMdP), Balcarce, Argentina. La investigación fue financiada por la UNMdP y el Instituto Nacional de Tecnología Agropecuaria (INTA), Argentina.

\section{Literatura citada}

Anónimo. 1992. La Culture du Tournesol. Guide Cultural. PROLEA-CETIOM, Centre Technique Ineterprofessionel des Oléagineaux Métropolotains (CETIOM). Paris, France. 30 pp.

Asagir. 2006. Red nacional de evaluación de cultivares comerciales de girasol. Cuadernillos informativos: Región Centro y Región Sur. Asociación Argentina de Girasol, Buenos Aires. http://www.asagir.org.ar. (Consultado: marzo de 2007).

Bartual, R., E. Carbonell, J. Marsal, J. Tello y T. Campos. 1991. Gene action in the resistance of peppers (Capsicum annuum) to Phytophthora stem blight (Phytophthora capsici L.). Euphytica 54:195-200.

Becker, W. 1985. Manual of Quantitative Genetics. Fourth ed. Academic Enterprises Pullman, Washington, USA. 181 pp.

Bertrand, F. y D. Tourvieille. 1987. Phomopsis du tournesol: tests de sélection. Informations Techniques du Cetiom 98:12-18.

Castaño, F. y M. Legarreta. 2004. Variabilidad de respuestas en girasol a las inoculaciones en el botón apical con Sclerotinia sclerotiorum. Journal of Basic and Applied Genetics 16 (Suplemento):117.

Castaño, F., F. Vear y D. Tourvieille. 1989. L'utilisation de plusieurs tests simultanés dans la sélection pour la résistance du tournesol visà-vis de Sclerotinia sclerotiorum. Information Techniques du Cetiom 107:14-20.

Castaño, F., F. Vear y D. Tourvieille. 1991. Sclerotnia sclerotiorum: les atouts de la lutte génétique chez le tournesol. p. 719-726, vol. 2. In: Proc. $3^{\text {rd }}$ International Conference of Plant Diseases, Bordeaux-Lac, France.

Castaño, F., F. Vear y D. Tourvieille. 1993. Resistance of sunflower inbred lines to various forms of attack by Sclerotinia sclerotiorum and relations with some morphological characters. Euphytica 68:85-98.

Castaño, F., F. Veary D. Tourvieille. 2001a. Horizontal resistances in sunflower: a review of a workshop at the $15^{\text {th }}$ International Sunflower Conference. Oléagineux, Corps Gras, Lipides 8:211-215.

Castaño, F., F. Vear y D. Tourvieille. 2001b. The genetics of resistance in sunflower capitula to Sclerotinia sclerotiorum measured by mycelium infections combined with ascospore tests. Euphytica 122:373-380.

Castaño, F., M. Hémery-Tardin, D. Tourvieille y F. Vear. 1992. The inheritance and biochemistry of resistance to Sclerotinia sclerotiorum leaf infections in sunflower (Helianthus annuus L.). Euphytica 58:209-219.

Degener, J., A. Melchinger, R. Gumber y V. Hahn. 1998. Breeding for Sclerotinia resistance in sunflower: A modified screening test and assessment of genetic variation in current germplasm. Plant Breeding 117:367-372.

Dorrel, D. y H. Huang. 1978. Influence of Sclerotinia wilt on seed-yield and quality of sunflower wilted at different stages of development. Crop Science 18:974-976.

Foley, D. 1969. Stalk deterioration of plants susceptible to corn stalk rot. Phytopathology 59:620-627.

García, C., K. Mulero, M. Agüero, F. Castaño, A. Ridao y R. Rodríguez. 2000. Sunflower performance after Phomopsis helianthi inoculation in the petiole. Test of Agrochemicals and Cultivars 21:17-18.

Godoy, M., F. Castaño, J. Ré y R. Rodríguez. 2005. Sclerotinia resistance in sunflower. I. Genotypic variations of hybrids in three environments of Argentina. Euphytica 145:147-154.

Gulya, T., K. Rashid y S. Masirevic. 1997. Sunflower diseases. p. 263-379. In: A. Schneiter (ed.). Sunflower technology and production. ASACSSA-SSSA, Wisconsin. USA.

Ingaramo, J. 2006. Plan estratégico Asagir 20062015. Cuadernillo Informativo ${ }^{\circ} 11$. ASAGIR (ed.), Buenos Aires, 23 pp.

Knowles, P. 1978. Morphology and anatomy. p. 5588. In: J. Carter (ed.). Sunflower Science and Technology. ASA-CSSA-SSSA, Wisconsin. USA.

Lamarque, C. 1985. Maladies et Accidents Culturaux du Tournesol. INRA, Paris. France. 120 pp.

Langar, K., Y. Griveau, F. Kaan, H. Serieys, D. Varès y A. Bervillé. 2002. Evaluation of parameters accounting for Phomopsis resistance using natural infection and artificial inoculation on recombinant inbred lines from a cross between 
susceptible and resistant sunflower. European Journal of Plant Pathology 108:307-315.

Micic, Z., V. Hahn, E. Bauer, A. Melchinger, S. Knapp, S. Tang y C. Schön. 2005. Identification and validation of QTL for Sclerotinia midstalk rot resistance in sunflower by selective genotyping. Theoretical and Applied Genetics 111:233-242.

Miller, J. 1987. Sunflower. p. 626-668. In: W. Fehr (ed.). Principles of Cultivar Development. MacMillan Pub. Co., NY, USA.

Odriozola, M., F. Castaño, A. Ridao, R. Rodríguez y J. Ré. 2006. Cuantificación de la podredumbre blanca de los capítulos en híbridos de girasol inscriptos en la Argentina durante el período 1980-1999. Revista de Investigaciones de la Facultad de Ciencias Agrarias-UNR (Argentina) 10:27-36.

Pérès, A., L. Allard y Y. Regnault. 1991. Sclerotinia sclerotiorum sur tige de tournesol: étude de la protection fongicide. Vol. 1, p. 393-400. In: Proc. $3^{\text {rd }}$ International Conference of Plant Diseases, Bordeaux-Lac, France.

Prats, E., M. Bazzalo, A. León y J. Jorrín, 2003. Accumulation of soluble phenolic compounds in sunflower capitula correlates with resistance to Sclerotinia sclerotiorum. Euphytica 132:321329.

Robert, N., F.Vear y D. Tourvieille, 1987. L'héredité de la resistance au Sclerotinia sclerotiorum (Lib.) de Bary chez le tournesol. I. Étude des réactions à deux tests mycéliens. Agronomie 7:423-429.
Sala, C., A. Vázquez, A. de Romano y S. Piubello. 1994. Sclerotinia mid-stalk rot in sunflower effect on yield components and oil content. Helia 17:41-44.

Schneiter, A. y J. Miller. 1981. Description of sunflower growth stages. Crop Science 21:901903.

Sposaro, M., P. Berry, M. Sterling, A. Hall y C. Climenti. 2007. Desarrollo y validación de un modelo para vuelco y quebrado en girasol. p. 308-309. En: Asagir (ed.) Actas del IV Congreso Argentino de Girasol, Buenos Aires. Argentina.

Steel, R. y J. Torrie. 1988. Bioestadística. Principios y Procedimientos. Second ed. Mc Graw-Hill, Bogotá. 622 pp.

Tourvieille, D. y F. Vear, 1990. Heredity of resistance to Sclerotnia sclerotiorum in sunflowers. III. Study of reactions to artificial infections of roots and cotyledons. Agronomie 10:323-330.

Vear, F. y D. Tourvieille. 1988. Heredity of resistance to Sclerotinia sclerotiorum. II. Study of capitulum resistance to natural and artificial ascospore infections. Agronomie 8:503-508.

Verschoor, M., F. Castaño, A. Ridao y R. Rodríguez. 1998. Phomopsis helianthi resistance in sunflowers after mid-stem inoculation. Annals of Applied Biology 132 (Supplement):48-49.

Zimmer, D. y J. Hoes. 1978. Diseases. p. 225262. In: J. Carter (ed.). Sunflower science and technology. ASA-CSSA-SSSA, Wisconsin. USA. 